\title{
Our experience with a single stage bilateral approach for treatment of bilateral middle cerebral artery aneurysms
}

\author{
A. Chiriac ${ }^{1,2}$, Georgiana Ion ${ }^{1}$, Z. Faiyad ${ }^{1}$, I. Poeata ${ }^{1,2}$ \\ 1 "Gr. T. Popa" University of Medicine and Pharmacy, Iasi, RomANIA \\ 2 "Prof. Dr. N. Oblu" Clinic Emergency Hospital, Iasi, ROMANIA
}

\begin{abstract}
Surgical management of bilateral middle cerebral artery (MCA) aneurysms is particular challenging clinical situation. For these patients various options of surgical treatment are available as unilateral approach, single stage bilateral craniotomy or two stage bilateral craniotomy. We report our experience with a case of bilateral MCA aneurysm which was managed by single stage bilateral pterional craniotomy.
\end{abstract}

\section{INTRODUCTION}

Surgical management of bilateral intracranial aneurysms is still difficult especially when it involves bilateral middle cerebral artery aneurysms. Even though endovascular treatment is widely accepted as the first option in the treatment of intracranial aneurysms, the middle cerebral artery aneurysms remained with quite high addressability for microsurgical clipping due to its special predisposition to have a broad neck that incorporate the parent vessels and frequent association with massive intracranial haemorrhages. Depending on some anatomical features, experience and preference of the neurosurgeon, these lesions can be operated in single or two separate sessions by unilateral or bilateral approach. Therefore, direct clipping of both ruptured and unruptured aneurysm in a single-stage operation, is more preferred today, if technically possible. The risk and worry about rupture of an untreated associated aneurysm is not insignificant during the early or late postoperative period when aggressive management against vasospasm is fundamental.

We report upon our experience with a single stage bilateral craniotomy approach for clipping the bilateral middle cerebral artery aneurysms.

\section{CASE REPORT}

A 49-year-old woman addressed to the emergency room with spontaneous severe headache, photophobia and few episodes of vomiting form one day. At clinical examination she was conscious with no motor deficits only slight nuchal rigidity. The cerebral CT-scan showed features of acute subarachnoid haemorrhage at basal cisterns

\section{Keywords \\ middle cerebral artery \\ aneurysm, \\ single stage, \\ bilateral approach}

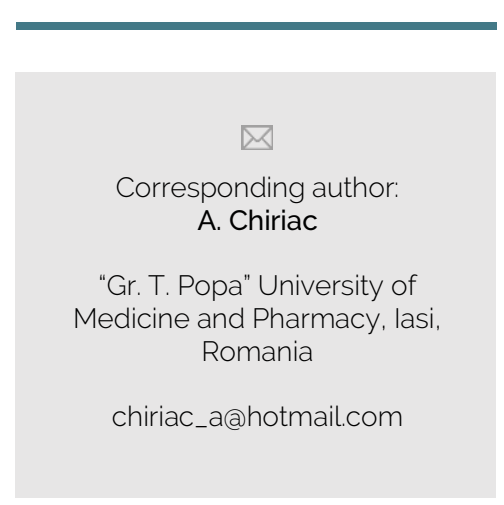

Copyright and usage. This is an Open Access article, distributed under the terms of the Creative Commons Attribution Non-Commercial No Derivatives License (https://creativecommons org/licenses/by-nc-nd/4.0/) which permits noncommercial re-use, distribution, and reproduction in any medium, provided the original work is

unaltered and is properly cited.
The written permission of the Romanian Society of The written permission of the Romanian Society of re-use or in order to create a derivative work.

ISSN online 2344-4959

(C) Romanian Society of Neurosurgery

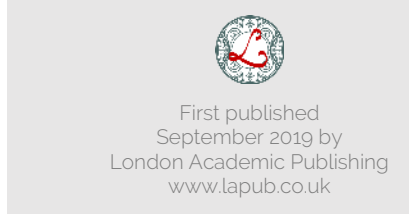


and both Sylvain fissure. The patient was classified as Fisher grade III and Hunt and Hess grade 1. CT angiography was performed and revealed bilateral MCA bifurcation aneurysms (Figure 1). The presence of a nipple on aneurysmal sac and the slight prevalent SAH in left Sylvian fissure suggested that the left was the ruptured aneurysm. Because the unruptured aneurysms at the contralateral MCA were not negligible and concerning represented by necessity of an aggressive triple $\mathrm{H}$ therapy a singlestage operation was decided. Single stage bilateral craniotomy and microsurgical clipping of the aneurysms was done in the same day of admission.

The procedure was performed with the patient under the general anaesthesia. A bilateral small sized pterional approach was performed in consecutive steps. The rupture side was approached first. The head is positioned on the operating table without being fixed in the frame with pins. The head is slightly rotated to the contralateral side and extended enough to minimize the frontal lobe retraction.

A curvilinear skin incision was made inside the hairline starting in front of the tragus ( $5 \mathrm{~mm}$ approx.) to the level of the midline. The incision on the attachment of the muscle to the superior temporal line is made with monopolar cut in order to detachment and retraction of the muscle posteriorly and inferiorly with hooks. A classic pterional craniotomy by tow blur holes is performed. By bone flap removal pterion bone and a prominent sphenoid wing are smoothed using a high-speed drill. Dura is C-like shape incised and reflected toward the cranial base. After minimal opening of the sylvian fissure, the optic, chiasmatic, and lamina terminalis cisterns are opened with gradually cerebrospinal fluid aspiration to obtain a slack brain and avoiding excessive retraction. With the highlighting of the internal carotid artery and its terminal bifurcation, microdissection is continued with widely opening of the sylvian fissure. A good exposure of the M1 segment is obtained. This segment is followed up to the bifurcation with careful microdissection of the aneurysmal neck to prevent the aneurysm rupture. After the clear exposure of the neck and the origins of the M2 branches a clip is applied with aneurysm exclusion. After carefully haemostasis and dura closure, the bone was fixed in two or three points. Finally the wound is closed as the usual fashion.
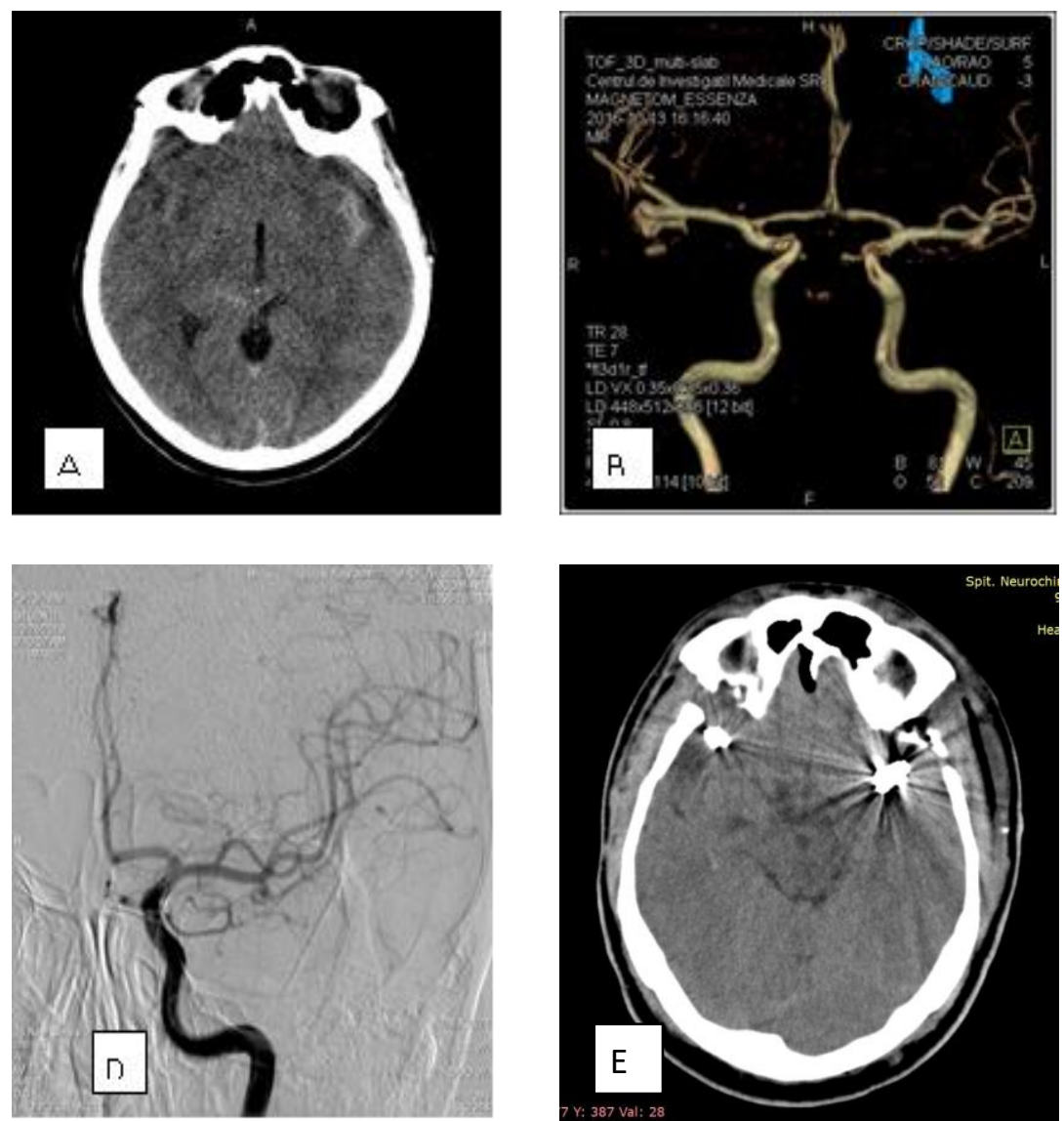

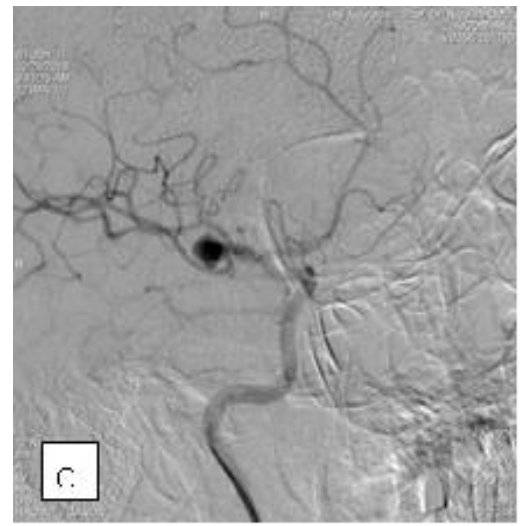

FIGURE 1.

A - Diagnostic CT-scan with left sylvian fissure haemorrhage;

B - 3D MRA;

C - DSA showing right MCA aneurysm;

D - DSA showing left ruptured MCA aneurysm;

E - Postoperative CT control. 
A total of three clips were used with two on the right side to preserve the bifurcation anatomy. The total time for surgery was four and a half hours. There was no intraoperative complication. Triple-H therapy was started immediately postoperatively. Control CT scan was performed 24 hours later. The patient evolution was favourable accompanied only by mild episode of headache. She was discharged home on the 21 th day.

\section{DISCUSSION}

The clinical status regarding the incidence of bilateral aneurysm of MCA was mentioned in the literature around $1.18 \%$. For bilateral MCA aneurysms, several surgical treatment options such as unilateral approach, single-stage bilateral craniotomy, or twosided bilateral craniotomy have been reported. For each type of approach it is extremely important to pay attention to several preconditions in order to perform them safely and effectively.[3,4,6,7]

Single stage surgery has the advantages of removing a new risk of bleeding, curative treatment with one-time risk of general anaesthesia, shorter hospital stay and reduced cost in terms of bed charge, investigations and operative expenditure.[3,4,6,7]

The most important reason for single stage surgery by bilateral approach is to eliminate the risk of bleeding from unruptured aneurysm in the early or late postoperative period. Two important large studies have showed that associated aneurysm in patients with SAH due to another ruptured aneurysm presents a higher risk for future bleeding than makes aneurysms of similar size with no SAH history $[3,4,6]$. Furthermore, the necessity of an aggressive hypertensive treatment for fighting with cerebral vasospasm may carry a potential risk of rupturing for associated untreated aneurysm in the postoperative period. $[4,6]$

Optimal selection of the patient with bilateral MCA aneurysm for a single stage surgery by unilateral or bilateral approach is dependent on a number of parameters such as severity of brain edema, the length of contralateral M1 segment, configuration and projection of the contralateral aneurysm. Presence of an important brain swelling, correlated with recent severe SAH is usually associated with very difficult microdisection and an excessive brain retraction that leads to a single stage bilateral approach choice. Longer contralateral M1 segment and a complex or large associated unruptured aneurysm are also condition this type of approach. If the anterior projection of the associated contralateral aneurysm is an indication for a unilateral single stage approach, the posterior or along the axis of the MCA projections are condition more favourable for a bilateral single stage approach.

Another important factor in bilateral MCA aneurysms surgery is represented by correct determining the side of ruptured aneurysm, because surgery should always be started from the ruptured side. In case of the misdiagnosed of ruptured bilateral MCA aneurysm with first clipping of unruptured one, the contralateral (ruptured) MCA aneurysm should always be clipped via a second contralateral craniotomy. A good clinical grade presentation can also be a factor favourable for a single stage bilateral surgery.

\section{CONCLUSIONS}

Challenging for a single stage bilateral approach should be considered in suitable cases of bilateral MCA aneurysm in all centres with experienced vascular neurosurgeons. A careful analysis based on clearly defined parameters will help the neurosurgeon in patient selection for this particular type of approach. This particular approach is more indicated in a patient with bilateral middle cerebral artery aneurysms presenting with bleeding and the ruptured side is not easily identifiable.

\section{REFERENCES}

1. Acik, V., Cavus, G., Bilgin, E., Arslan, A., Gezercan, Y., \& Okten, A. I. (2017). Surgical treatment of mirror middle cerebral artery aneurysms: bilateral and unilateral approach. World neurosurgery, 108, 774-782.

2. Andrade-Barazarte, H., Kivelev, J., Goehre, F., Jahromi, B. R., Noda, K., Ibrahim, T. F. \& Hernesniemi, J. A. (2015). Contralateral approach to bilateral middle cerebral artery aneurysms: comparative study, angiographic analysis, and surgical results. Neurosurgery, 77(6), 916-926.

3. Inci, S., Akbay, A., \& Ozgen, T. (2012). Bilateral middle cerebral artery aneurysms: a comparative study of unilateral and bilateral approaches. Neurosurgical review, 35(4), 505-518.

4. Maruyama, Keisuke \& Kurita, Hiroki \& Yamaguchi, Ryuichi \& Noguchi, Akio \& Shiokawa, Yoshiaki. (2013). One-Stage Clipping of Bilateral Middle Cerebral Artery Aneurysms Via 
the Bilateral Pterional Keyhole Approach. Neurologia medico-chirurgica. 53. 148-52. 10.2176/nmc.53.148.

5. Rajagopal, N., Balaji, A., Yamada, Y., Kawase, T., \& Kato, Y. (2019). Etiopathogenesis, clinical presentation and management options of mirror aneurysms: A comparative analysis with non-mirror multiple aneurysms. Interdisciplinary Neurosurgery, 18, 100535.
6. Shin BG, Kim JS, Hong SC. (2005). Single-stage operation for bilateral middle cerebral artery aneurysms. Acta Neurochir (Wien). 2005 Jan;147(1):33-8; discussion 38.

7. Yam B Roka, Manzil Shrestha. (2013). Single Stage Clipping of Bilateral Middle Cerebral Aneurysms. Nepal Journal of Neuroscience vol 2, 10:86-87. 